\title{
Temperature dependences of SnTe linear expansion coefficient
}

\author{
E.I. Rogacheva ${ }^{1}$, V.P. Popov ${ }^{2}$, O.N. Nashchekina ${ }^{1}$ \\ ${ }^{1}$ National Technical University "Kharkov Polytechnic Institute", 21 Frunze st., 61002 Kharkov, Ukraine \\ ${ }^{2}$ Kharkov National University, 4 Svoboda Sq., 61077 Kharkov, Ukraine
}

\begin{abstract}
The influence of kinetic factors on the temperature dependences of the linear expansion coefficient $(\alpha)$ for SnTe crystals with different degrees of deviation from stoichiometry was studied. The $\alpha(T)$ dependences were obtained by using stationary and dynamic regimes. In the case of the stationary regime, an increase in $\alpha$ with increasing temperature $(4.2-300 \mathrm{~K})$ was registered, and anomalies in the $\alpha(T)$ dependences were observed and attributed to phase transitions. After quick plunging into liquid nitrogen and subsequent heating the samples up to $300 \mathrm{~K}$ without keeping them for a long time at fixed temperatures (a dynamic regime), the $\alpha(T)$ dependences exhibited an oscillatory behavior, most pronounced in the sample with 50.4 at.\% Te. It is suggested that the observed behavior of the $\alpha(T)$ dependences is connected with an oscillatory process of approaching the equilibrium in the intrinsic defect subsystem and with overlapping of relaxation processes and temperature phase transitions.
\end{abstract}

Keywords: tin telluride, nonstoichiometry, native defects, phase transition, coefficient of linear expansion, temperature dependences, kinetic factors, relaxation processes.

Paper received 04.09.02; accepted for publication 17.12.02.

\section{Introduction}

Thermal expansion is an important physical characteristic, which in a number of cases determines the possibility to practically use investigated material, in particular for IR applications. Studying the temperature dependences of the coefficient of linear expansion is a highsensitivity method to detect phase transitions.

The object of the study is SnTe, a IV-VI semiconducting compound $[1,2]$, which has a wide $(\sim 1$ at. $\%)$ singlesided homogeneity region located on the side of excess $\mathrm{Te}$ (the peak in melting curves in the Sn-Te system corresponds to 50.4 at. \% Te) [2,3]. It is well known that $\mathrm{SnTe}$ undergoes a ferroelectric displacive phase transition $[4,5]$ at the temperature $T_{c}$ close to $100 \mathrm{~K}$. The transition is accompanied by the change in crystal lattice symmetry from cubic to rhombohedral and the appearance of anomalies in the temperature dependences of different physical properties. The existence of the structural phase transition is usually attributed to the softening of the transverse optical mode as a result of electron-phonon interactions. It was found that growth in charge carrier concentration leads to a decrease in $T_{c}$, and at 50.4 at. \% Te this phase transition disappears [4-7].
SnTe is a very convenient object for studying the influence of vacancies on physical properties of crystals due to a high ( $\sim 1-2$ at. $\%)$ concentration of non-stoichiometric cation vacancies. In particular, the high concentration of vacancies determines the possibility of their interactions, which, in turn, can stimulate the ordering of vacancies. On the other hand, the high concentration of cation vacancies must lead to the specificity of relaxation processes, which occurs in the system deviated from the equilibrium state.

Earlier we reported on observing new, previously unknown temperature phase transitions in crystals and thin films of SnTe with a high degree of deviation from stoichiometry using X-ray diffractometry and transport property measurements [8-10]. We suggested that the transitions were related to redistribution of defects at their sufficiently high concentrations and formation of defect configurations corresponding to the free energy minimum at a given temperature. Using low-temperature (77-300 K) $\mathrm{X}$-ray diffraction analysis, we detected two phase transitions in non-stoichiometric tin telluride in the temperature ranges of $135-150$ and 200-215 K [10], which were most pronounced for the sample with 50.4 at. \% Te. A substantial magnitude of the effects and a decrease in the unit cell parameter with increasing temperature in cer- 


\section{E.I. Rogacheva et al.: Temperature dependences of SnTe ...}

tain ranges (that is negative a), accompanying the phase transitions in the sample with 50.4 at. \% Te, were attributed to the influence of kinetic factors, such as non-equilibrium initial state of samples resulting from quick cooling down to $77 \mathrm{~K}$ and relaxation phenomena taking place during heating. It was also established that the magnitude of the effects accompanying the phase transition decreases under increasing the degree of deviation from stoichiometry. It was explained in [10] as a result of an increase in the diffusion rate with growing concentration of cation vacancies. A question arises whether the observed phase transitions are equilibrium and real or they are of purely relaxation nature. That is why it seemed important to clarify how kinetic factors can affect the manifestation of the anomalies in the temperature dependences of properties.

To answer this question, in the present work, a dilatometry study was carried out on tin telluride samples with Te contents ranging from 50.4 to 51.2 at.\% Te, the temperature dependences of the linear expansion coefficient $(\alpha)$ being obtained under two different regimes. The first regime was close to quasi-equilibrium conditions, the other corresponded to apparently non-equilibrium conditions of heating. As a result, the existence of phase transitions in tin telluride was proved using the dilatometry method. It was also established that the behavior of the $\alpha(T)$ dependences essentially depends on kinetic factors, and under non-equilibrium conditions the process of returning to the equilibrium state under heating has an oscillatory character. This oscillatory behavior of relaxation processes was attributed to a high concentration of non-stoichiometric defects leading to loosening of the structure.

\section{Experimental}

The samples were synthesized in evacuated down to $10^{-2}$ Pa quartz ampoules at $1200 \mathrm{~K}$ during 5-6 hours under vibrational mixing of the melt. Then the samples were subjected to homogenizing annealing at $T=820 \mathrm{~K}$ and subsequent cooling down with the switched-off furnace. Under this thermal treatment the homogeneity region of SnTe is usually within 50.1-50.8 at.\% Te [2,3]. The compositions of the studied samples corresponded to 50.4, $50.5,50.7$, and 51.2 at. $\%$ Te. The temperature dependencies of the coefficient of linear expansion in the temperature range of $5-300 \mathrm{~K}$ or $77-300 \mathrm{~K}$ were obtained using a differential dilatometer with optical-mechanical registration of thermal elongation on the samples prepared by hot pressing from polycrystalline bulks. The stationary regime consisted in cooling samples down to the temperature of liquid helium slowly and then heating the samples up to room temperature with keeping them at measurement temperatures until complete temperature stabilization is reached. Under the dynamic regime samples were quickly plunged into liquid nitrogen, and the $\alpha(T)$ curves were recorded under heating.

\section{Results and discussion}

In the case of a stationary regime, an increase in a with increasing temperature was registered, and anomalies in the $\alpha(T)$ curves were observed. The initial increase in a is connected with the temperature dependence of the coefficient of linear expansion which continues up to $\sim 130$ $140 \mathrm{~K}$ (the Debye temperature of SnTe [2]). In the $\alpha(T)$ curve of the sample with 50.4 at.\% Te (Fig. la), three effects in the temperature ranges of 100-110 K, 140-150 K, and 190-200 K were observed. The $\alpha(T)$ curves for the samples with 50.7 and 51.2 at.\% Te have an inflexion point at $\sim 100-110 \mathrm{~K}$ and plateaus at 180-190 K (Fig. 1b). It is natural to attribute these anomalies to phase transitions taking place in SnTe crystals and thin films with a significant degree of deviation from stoichiometry. In [8-10], only two temperature anomalies of the unit cell parameter at $135-150$ and $200-210 \mathrm{~K}$ were reported.

When measurements were carried out in a dynamic regime, the behavior of the $\alpha(T)$ curves changed significantly for all studied compositions (Figs 1,2). Usually a sharp initial increase in $\alpha$ in contrast with the stationary value was observed and under the subsequent heating, the $\alpha(T)$ dependences exhibited either an oscillatory character (for the samples with 50.4, 50.5, and 50.7 at.\% Te) or a monotonic decrease in $\alpha$ with increasing temperature (for the samples with 51.2 at. \% Te). An oscillatory behavior was most pronounced in the sample with 50.4 at. $\%$ Te. In the $\alpha(T)$ curve for this sample, distinct minima at $\sim 110 \mathrm{~K}$ and $\sim 200 \mathrm{~K}$ were observed (Fig. la). The magnitude of these effects decreases regularly with increasing Te concentration (Fig. 2). The $\alpha(T)$ dependences for the samples with 50.5 at.\% Te and 50.4 at.\% Te behave similarly, while in the $\alpha(T)$ curve of the sample with 50.7 at. $\%$ Te in the vicinity of $110 \mathrm{~K}$, an additional oscillation is registered (Fig. 2). In the sample containing 51.2 at. $\%$ Te, which is outside the homogeneity region, the equilibrium state is approached monotonically. Note that for all studied compositions, starting from $220-240 \mathrm{~K}$, the curves obtained in stationary and dynamic regimes virtually coincided (Figs 1,2).

As is seen from the obtained data, the behavior of the $\alpha(T)$ dependences essentially depends on regimes, under which they were measured. The $\alpha(T)$ curves recorded under a stationary regime using the dilatometry method not only prove the existence of phase transitions at $135-150 \mathrm{~K}$ and 200-210 K [8-10] for the sample with 50.4 at.\% Te, but also exhibit a peculiarities at $100-110 \mathrm{~K}$. With increasing Te content, the effect at 135-150 K disappears.

Analyzing the obtained data, one can suggest that due to quick cooling of samples plunged into liquid nitrogen, they get oversaturated with defects, whose concentration exceeds the equilibrium concentration. The processes of defect redistribution are not finished and ordered configurations corresponding to low temperatures are not formed. A significant deviation from the equilibrium and oversaturation with defects are proved by the sharp increase in a measured in the dynamic regime as compared 
E.I. Rogacheva et al.: Temperature dependences of SnTe ...
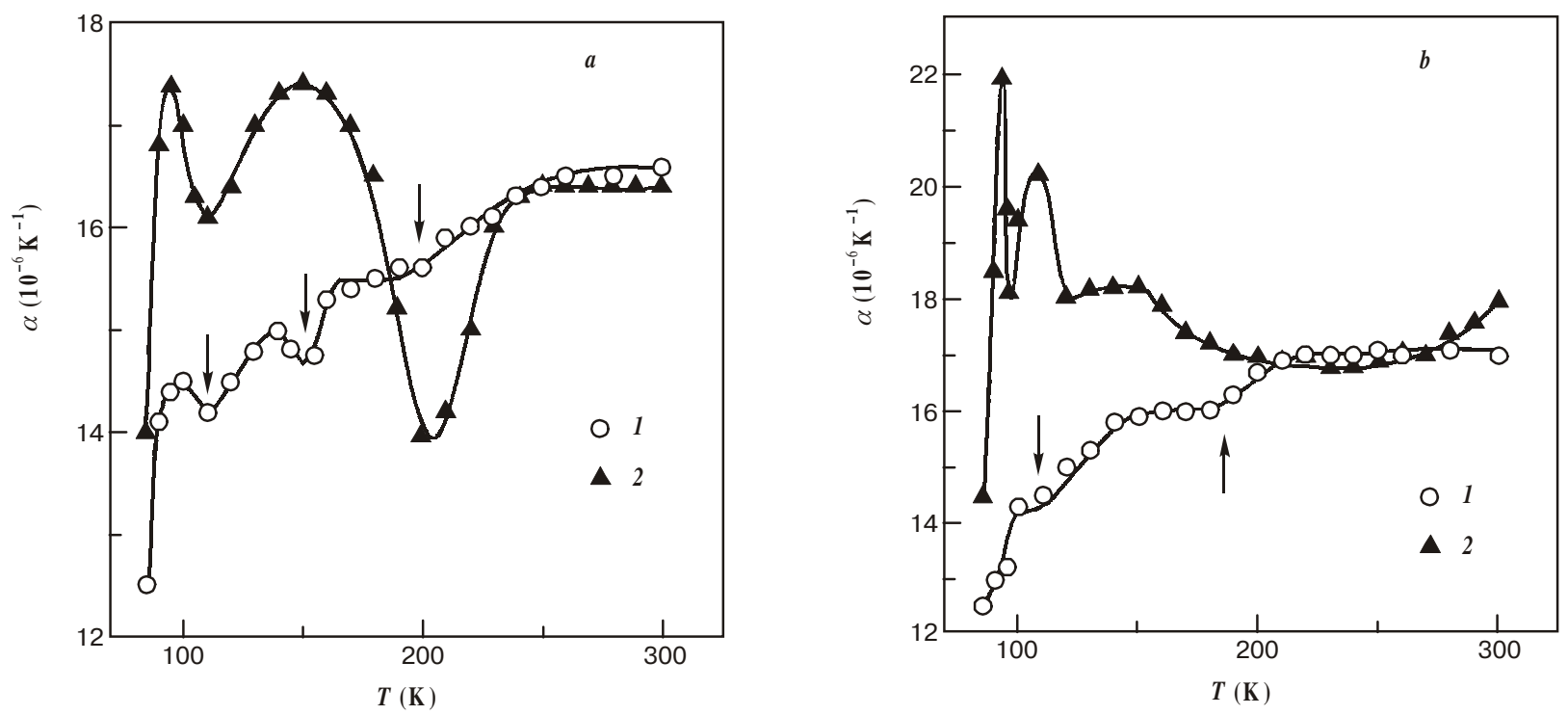

Fig. 1. Temperature dependences of the thermal expansion coefficient for tin telluride with (a) 50.4 and (b) 50.7 at. $\%$ Te: $1-$ stationary regime; 2 - dynamic regime.

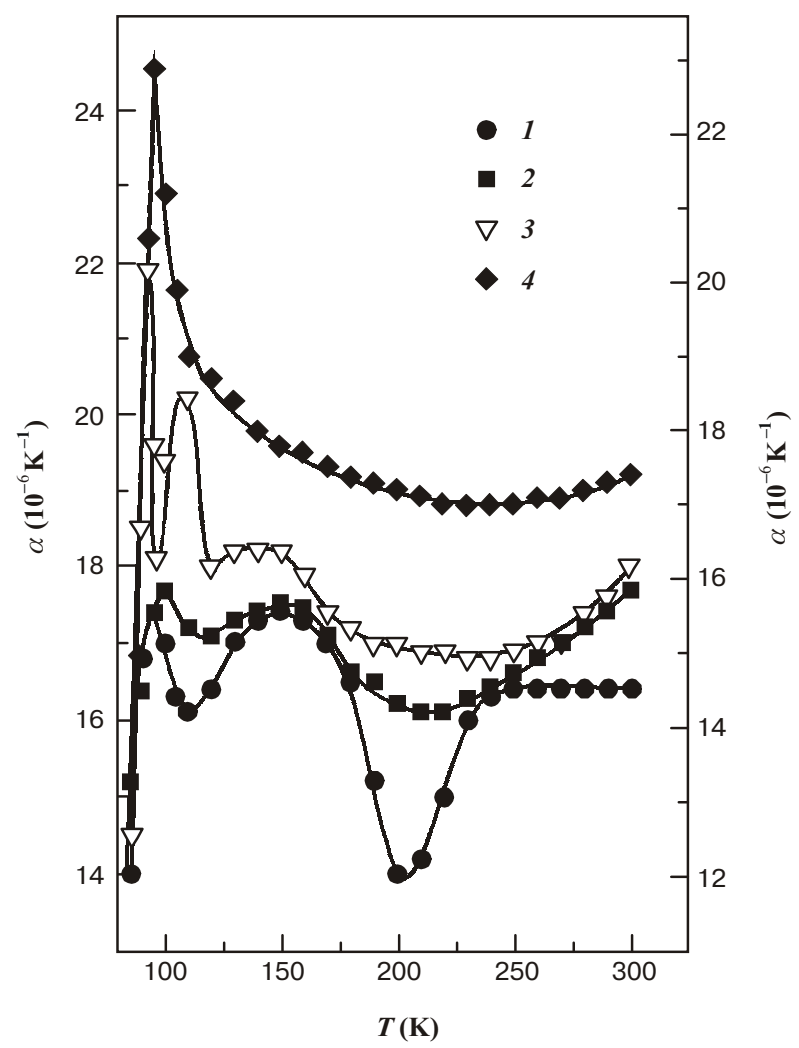

Fig. 2. Temperature dependences of linear expansion coefficient for tin telluride with different deviation from stoichiometry obtained in the dynamic regime: $1-50.4 ; 2-50.5 ; 3-50.7 ; 4-$ 51.2 at. $\% \mathrm{Te}$.

to $\alpha$ values obtained in the stationary regime, which is manifested clearly in all samples (Fig. 1,2). Indeed, the abatement of quasi-elastic bond and increase in the crystal anharmonicity occurring under the crystal disorder- ing due to its oversaturation with defects should lead to an increase in $\alpha$. With increasing concentration of vacancies (increasing Te concentration) a growth in $\alpha$ as a result of plunging the sample into liquid nitrogen becomes more significant, which indicates an increase in the degree of disordering in the system.

Under heating of such a non-equilibrium system, due to an increase in the diffusion rate, which leads to defect movement (migration) and partial annihilation of defects, complex processes should take place. These processes are caused on the one hand, by the relaxation of the system towards the equilibrium state and decreasing in $\alpha$ and, on the other hand, by phase transformations which occur in a system far from the equilibrium state. Such processes can result in gigantic lattice instability in the vicinity of the temperature of a phase transition (equilibrium or non-equilibrium). When returning to the equilibrium state the system can pass through a number of intermediate states corresponding to metastable equilibria at a given temperature. As a result, the character of the $\alpha(T)$ dependences becomes more complex. The fact that values of a under the two described regimes coincide at temperatures above $\sim 240 \mathrm{~K}$ shows that in the vicinity of this temperature the system returns to the equilibrium, and the state of sample defect subsystem is identical for both regimes.

As is seen from Fig. 2, the character of the relaxation processes and kinetics of equilibrium setting in the crystal defect subsystem depend on the sample composition, i.e. on cation vacancy and charge carrier concentrations. As follows from the obtained data, the $\alpha(T)$ dependences for most of the studied samples have an oscillatory behavior, although locations, amplitudes and nature of oscillations are different. Oscillations with minima at 100-110 and 190-200 K are most pronounced for the sample with 50.4 at.\% Te, and with increasing Te con- 


\section{E.I. Rogacheva et al.: Temperature dependences of SnTe ...}

tent, these oscillations become less distinct (Fig. 2). One can suggest that these oscillations are associated mainly with the defect redistribution processes (phase transitions) taking place under non-equilibrium conditions.

The composition with 50.4 at. \% Te, corresponding to maxima in the liquidus and solidus curves in the $\mathrm{Sn}-\mathrm{Te}$ [1-3] system, represents one of the optimum compositions for vacancy ordering. A simple estimation [3] shows that this composition corresponds to the formation of a f.c.c. lattice of vacancies with the parameter $a=4 a_{0}$, where $a_{0}$ is the SnTe unit cell parameter. The fact that not only maximum in melting curves but also peculiarities in the concentration dependences of some properties [3,11-13] within the $\mathrm{SnTe}$ homogeneity region correspond to 50.4 at.\% Te can be considered as an argument in favor of ordering. That is why oscillations observed in the $\alpha(T)$ curve of the sample with 50.4 at.\% Te measured under a dynamic regime (Fig. la), can be caused to a great extent by the redistribution of nonstoichiometric defects, which takes place in a system far from the thermodynamic equilibrium state. A larger magnitude of the effect at $190-200 \mathrm{~K}$ in the sample with 50.4 at. $\%$ Te as compared to the stationary regime is apparently connected with this circumstance.

For the samples with 50.5 and 50.7 at.\% Te the $\alpha(T)$ dependences are similar but the effect manifestation is less distinct. The most probable reason for that is deviation from the optimum ordered composition and, as a result, diminishing of stimuli for ordering. However, an increase in a comparing to its equilibrium value becomes more noticeable, and an oscillatory character of relaxation processes manifests more distinctly. In particular, in the $\alpha(T)$ dependence of the sample with 50.7 at.\% Te, an additional oscillation near $100 \mathrm{~K}$ is observed. In the sample with 51.2 at. $\%$ Te, the relaxation curve exhibits monotonic behavior. It is possible that the presence of fine Te inclusions in this sample, whose composition is outside the homogeneity region, increases resistance of the crystal lattice and thus leads to an increase in the oscillation period.

Thus, the effects connected with the phase transitions are most intensive in the sample with 50.4 at.\%Te, and with increasing Te content they manifest less distinctly. For the composition with 50.7 at.\%Te, the process of approaching the equilibrium in the intrinsic defect subsystem has an oscillatory character, whereas at 51.2 at. \%Te the system returns monotonically into the equilibrium state.

To all appearances, an oscillatory character of relaxation processes is connected to a great extent with a high concentration of non-stoichiometric cation vacancies, presence of which leads to loosening the crystal lattice, slackening bonds and decrease in the period of oscillations, thus making them observable. If a system is driven out of the equilibrium, the probability of oscillations increases under sufficiently high susceptibility of the system to these oscillations and reasonably low damping factor. One can suggest that a high concentration of vacancies stimulates oscillations making the system more "mobile" and sensitive to external effects, such as "heat strokes" as a result of quick cooling.
In [10], under a dynamic regime, pronounced anomalies in the unit cell parameter were observed at 135-150 and $200-210 \mathrm{~K}$. The anomalies were most distinct for the sample with 50.4 at.\% Te, for which even negative values of $\alpha$ (a drop in the unit cell parameter under increasing temperature) were reported. In the present work, we did not observe negative values of $\alpha$, however in the vicinity of $200 \mathrm{~K}$, a sharp decrease in $\alpha$ was registered. The difference between values of $\alpha$ obtained in [10] and in the present study is apparently connected with different techniques of sample preparation. In [10] powder samples were used, whereas in this work, bulk crystals prepared by hot pressing and subsequent annealing were studied. One can suggest that for the former, due to their small volume, quenching by plunging into liquid nitrogen proved more effective and led to a higher level of saturation with defects.

\section{Conclusions}

The temperature dependences of the linear expansion coefficient were obtained using the dilatometry method under different regimes for SnTe samples with various degrees of deviation from stoichiometry (50.4-51.2 at.\% $\mathrm{Te})$. On the basis of the study, the existence of equilibrium phase transitions at 140-150 and 190-200 K, which was detected earlier using X-ray diffractometry and measurements of galvanomagnetic properties in SnTe crystals as well as thin films with Te excess was proved. An additional phase transition at $100-110 \mathrm{~K}$, which was not registered before in $\mathrm{SnTe}$ with a high degree of deviation from stoichiometry was observed. It is suggested that all above mentioned phase transitions are caused by the processes of redistribution of non-stoichiometric defects.

A significant influence of kinetic factors (rate of quenching, rate of heating and duration of keeping samples at a fixed temperature in the process of heating) on the $\alpha$ temperature dependences was detected. Quick plunging of samples into liquid nitrogen leads to fixation of non-equilibrium states, an increase in the degree of crystal anharmonicity and consequently to an increase in $\alpha$. Under subsequent rapid heating the behavior of the $\alpha(T)$ dependences becomes complex, which reflects overlapping of relaxation processes and phase transitions taking place in a system far from the equilibrium state. The observed oscillatory behavior of relaxation processes is related to loosening of the lattice caused by a high concentration of cation vacancies. It is shown that starting from $220-240 \mathrm{~K}$, the state of the system approaches the equilibrium, irrespective of the regime of measuring the $\alpha(T)$ dependences.

It follows from the obtained data that studying of phase transitions caused by the redistribution of nonstoichiometric defects over the cation sublattice should be conducted under strictly equilibrium conditions. To all appearances, the effects observed are characteristic for compounds with a high defect concentration, and $\mathrm{SnTe}$ is one of their typical representatives. 


\section{E.I. Rogacheva et al.: Temperature dependences of SnTe ...}

\section{Acknowledgments}

This work was supported by the Ministry of Education and Science of Ukraine

\section{References}

1. A.V. Lyubchenko, E.A. Salkov, F.F. Sizov, Physical principles of semiconductor infrared photoelectronics, Naukova Dumka, Kiev, 1984 (in Russian).

2. N.H. Abrikosov and L.E. Shelimova, Semiconducting materials based on $A^{4} B^{6}$ compounds, Nauka, Moscow, 1975 (in Russian).

3. E.I. Rogacheva, G.V. Gorne, N.K. Zhigareva, and A.I Ivanova, Homogeneity region of tin monotelluride // Inorganic Materials, 27(2), pp. 194-197, 1991.

4. P.B. Littlewood, Phase transitions and optical properties of IV-VI compounds // Lect. Notes Phys., 152, pp.238-246, 1982.

5. T. Suski, Phase transitions and resistivity anomalies in $(\mathrm{Pb}$, Sn, Ge)Te compounds // Mater. Sci., 11(3), pp.3-53, 1985.

6. K.L. Kobayashi, Y. Kato, Y. Katayama, and K.F. Komatsubara, Carrier-concentration-dependent phase transition in SnTe // Phys.Rev.Letters, 37(12), pp.772-774, 1976.
7. S. Sugai, K. Murase, S. Katayama, S. Takaoka, S. Nishi, and H. Kawamura, Carrier density dependence of soft TP-phonon in SnTe by Raman scattering // Sol.St.Communs, 24(5), pp.407-409 (1977)

8. O.N. Nashchekina, E.I. Rogacheva, Temperature anomalies of transport properties in SnTe epitaxial thin films // Physica Status Solidi, 169(2), p.235-238 (1998).

9. O.N. Nashchekina, E.I. Rogacheva, A.I. Fedorenko, Phase transitions and transport properties in the SnTe crystals and thin films // Proceedings of SPIE, 3890, pp. 363-369 (1999).

10. O.N. Nashchekina, E.I. Rogacheva, A.I. Fedorenko, A.P. Isakina, and A.I. Prokhvatilov, Low-temperature lattice instability in SnTe // Low Temp. Phys., 25, pp. 285-289 (1999).

11. E.I. Rogacheva, N.A. Sinelnik, O.N. Nashchekina, V.P. Popov, T.A. Lobkovskaya, Defects of non-stoichiometry and dynamic stability of SnTe crystal lattice // Acta Physica Polonica A., 84(4), p. 733-736 (1993).

12. O.N. Nashchekina, E.I. Rogacheva, L.P. Shpakovskaya, V.I. Pinegin, A.I. Fedorenko, Non-stoichiometric defects in semiconductor SnTe thin films // Material Research Society Symposium Proceedings, 378, p.255-260 (1995).

13. E.I. Rogacheva, G.V. Gorne, O.N. Nashchekina, Deviation from stoichiometry and lattice properties of semiconducting SnTe phase // Material Research Society Symposium Proceedings, 378, p.107-1 12 (1995). 